

\section{Leer la ciudad: una apuesta para aprender una geografía viva} Reading the City: A Bet to Learn a Geography Alive Ler a cidade: uma aposta para aprender uma geografia viva

Jorge Isaac Ramírez Acosta*

Nathalia Zapata Rivera**

\section{Resumen}

Acercarse al concepto de ciudad implica múltiples retos, uno de ellos es la diversidad de acepciones que se encuentran al respecto al tratarse de un concepto inter y transdisciplinar. Así, en este trabajo se hace una aproximación al lenguaje sobre la ciudad en las ciencias sociales y en la didáctica de la geografía para identificar elementos que permiten la incorporación de la ciudad en el currículo, con la finalidad de hacer de la enseñanza una práctica viva que brinde conocimientos socialmente útiles a los estudiantes en su cotidianidad a partir del diseño de estrategias pedagógicas contextualizadas.

\section{Palabras clave:}

Ciudad, didáctica de la geografía, enseñanza renovada. 


\begin{abstract}
Approaching the concept of the city implies several challenges, one is the diversity of the meanings found in this regard because it is an inter and transdisciplinary concept. It is done an approach to the language about the city in social sciences, and later in geography teaching. It is intended to identify elements that allow the city incorporation in the curriculum, in order to make teaching a live practice that provides students with useful social knowledge in their daily lives, from the design of contextualized pedagogical strategies.
\end{abstract}

\section{Resumo}

Abordar o conceito de cidade implica múltiplos desafios, um deles é a diversidade de significados encontrados a esse respeito, toda vez que, é um conceito inter e transdisciplinar. Assim, neste trabalho é feita uma abordagem à linguagem sobre a cidade nas ciências sociais e, posteriormente, na didáctica da geografia, para identificar elementos que permitam a incorporação da cidade no currículo, a fim de tornar o ensino uma prática viva que forneça conhecimentos socialmente úteis nas sua vida diária a partir do desenho de estratégias pedagógicas contextualizadas.

\section{Keywords:}

City, didactics of geography, renewed teaching.

\section{Palavras-chave:}

Cidade, didáctica da geografia, ensino renovado. 


\section{Introducción}

La palabra ciudad ha sido usada históricamente por diversos actores para definir una multiplicidad de fenómenos y espacios con características particulares, lo que ha dado lugar a una adjetivación del término para hacer énfasis en una parte de la realidad que se pretende conocer y, en consecuencia, poder hablar de ciudad turística, ciudad capital, global, región innovadora, educada, violenta, entre otras. De este modo, "una palabra se ajusta a la otra atribuyendo a la ciudad significados adicionales o diferentes" (Pereira, 2007, p. 75), lo que en primera y última instancia lleva a una reducción del término en la medida que este se define a partir del desarrollo de una actividad económica, del devenir histórico, de la concentración de poder o incluso de la humanización de la ciudad. Llorente (2014) afirma:

La ciudad está hecha de fragmentos y se multiplica en tantas formas de posibilidad que, en su extremo, puede terminar disuelta en las fracciones de los miles de nombres que designan las ciudades del mundo, las poblaciones y los lugares habitados. (p. 13).

Si bien es claro que "no existe un lenguaje capaz de atravesar la totalidad del fenómeno espacial, de su conciencia y experiencia, de su conocimiento y representación, ni siquiera acotándolo dentro del ámbito de la ciudad" (Llorente, 2014, p. 12), es necesario revisar detenidamente el conjunto de elementos teóricos que lo conforman para hacer un filtro sobre los cambios y permanencias en las aproximaciones existentes sobre la ciudad, de tal manera que no todo sea válido, sino que se reconozcan los elementos comunes dentro del vasto universo de conocimientos científicos. Es por ello que, para comprender los fenómenos, problemas, posibilidades y necesidades en contextos particulares, los maestros deben orientar los procesos de enseñanza y aprendizaje desde las dinámicas de los territorios en los que viven los estudiantes.

Ahora bien, el salto de lo cuantitativo a lo cualitativo en la ciencia dio lugar a toda una serie de elaboraciones sobre la ciudad en las que se otorgó voz a los sujetos y en la que esta aparece como una construcción social que, de acuerdo con Aranguren (2000), es "donde se objetivan y apropian mutuamente lo material y lo simbólico” (p. 539), planteamiento que reivindica la importancia de las lecturas que realizan los sujetos del espacio vivido. Desde esta postura epistemológica

no es posible acceder al conocimiento del objeto ciudad, obviando las condiciones de vida del ser humano que la habita, como tampoco será dado entender la existencia humana separada de la sociedad, de la historia y de la cultura que definen el movimiento urbano. (Aranguren, 2000, p. 542).

Al ser la ciudad una invención humana está sujeta a cambios que reflejan los intereses de los múltiples actores que la habitan, por ello "un proyecto de vida contiene un proyecto de ciudad, así como un proyecto de ciudad comprende un proyecto de vida" (Aranguren, 2000, p. 543). Desde esta perspectiva, las transformaciones no son solo producto de los momentos históricos de las sociedades en términos políticos, económicos, sociales y culturales, sino fruto de las interacciones que en ella tienen lugar y que reflejan la existencia de la diferencia y la heterogeneidad, la cual va más allá del plano material y trasciende al de las representaciones y construcciones simbólicas.

\section{La ciudad desde las ciencias sociales}

La palabra ciudad proviene del latín civitas que representa un área urbana de alta densidad poblacional. Puede que se haya usado desde el Neolítico (Edad de Piedra) con las primeras aglomeraciones de población en un solo espacio (Herce, 2013) hasta muchos siglos después, puesto que la concentración demográfica se constituyó en el elemento clave del concepto. Con vigencia hasta la actualidad, debido al denominado proceso de urbanización, la concentración demográfica transformó progresivamente la estructura rural fruto de los movimientos migratorios causados por factores económicos, catastróficos o socioculturales.

En la historia clásica de carácter lineal, la ciudad se define a partir de cinco periodos y denominaciones tradicionales (antigua, medieval, moderna, industrial y posindustrial). Esta clasificación que, además obedece a un criterio de temporalidad, sienta las bases para entender el desarrollo de las ciudades como una sucesión de etapas predeterminadas, implica que los procesos sociales que en ellas tienen lugar sean entendidos a la luz de leyes similares a las de los fenómenos naturales. La ciudad antigua aparece en la historia como el primer lugar donde se asentaron los grupos humanos caracterizados por el sedentarismo y el comercio, hecho que supuso la desaparición parcial de las sociedades de cazadores y recolectores. Por su parte, la ciudad medieval surge como el espacio por excelencia en el que vive la burguesía rodeada de murallas, dando paso a un nuevo sistema de organización política en el cual el soberano es responsable de agenciar su desarrollo. Después, la ciudad moderna hace del espacio un lugar que se debe planificar y conquistar mediante el diseño de construcciones, en ella los desarrollos tecnológicos generaron la demanda de mano de obra para el ejercicio de diversas actividades económicas. La cuarta ciudad surge como producto de la revolución industrial, albergando las fábricas y potencializando el desarrollo de viejos y nuevos medios de transporte, mientras la quinta se caracteriza por el desarrollo de actividades económicas que se inscriben en el sector terciario (servicios) que conllevan a la producción de bienes no materiales.

Los elementos constitutivos de la tipología de ciudad presentada anteriormente muestran la dificultad que existe no solo para nombrar esta construcción humana, sino para reconocer los procesos que en ella se 
desarrollan de manera integral. De ahí que no sea posible su conocimiento en sentido amplio mediante su adjetivación, ya que una ciudad puede ser muchas a la vez por sus características y dinámicas y en esencia ser solo una por su configuración particular que la hace única. No obstante, los procesos sociales que tienen lugar en los distintos grupos humanos hacen posible pensar la ciudad como un complejo sistema dinámico, en el cual las formas espaciales y las acciones de las personas se encuentran en continua interacción. De acuerdo con David Harvey (1977) existe una relación constante entre el espacio y los sujetos, hecho que hace posible una organización espacial que refleja el pensamiento de las sociedades y que no puede ser negada. En tal sentido la ciudad deja de ser un espacio per se que va más allá de lo material y se configura a partir de las interacciones sociales y del sentido que los sujetos le otorgan.

Para Brousseau (citado por Alderoqui, 2002, p. 40), la ciudad es "un macroespacio [...] cuya dimensión solo puede abarcarse a través de una sucesión de visiones locales, separadas entre sí por desplazamientos del sujeto sobre la superficie terrestre". En tal sentido, al interior de las ciudades existen múltiples realidades que reflejan las diferentes situaciones, condiciones, problemas y posibilidades de quienes las habitan, de ahí que no sea posible realizar generalizaciones sobre los fenómenos que en ellas se presentan. Así, la ciudad emerge como un caleidoscopio en el cual se puede reconocer la realidad como heterogénea y variable, permitiendo múltiples miradas en relación con sus dinámicas. Santos (1990), a su vez, aborda la ciudad desde la transformación de la naturaleza por las acciones humanas a lo largo del tiempo, de ahí que la explique a partir de la noción de rugosidades. Las ciudades como un espacio socialmente construido a partir de las prácticas y procesos de los actores sobre los objetos, reflejan la forma como históricamente han sido planificadas de acuerdo con las lógicas de producción y distribución de la economía capitalista, motivo por el cual es posible observar espacios cuyo uso residencial, comercial, industrial, de servicios o de usos especiales es definido previamente en función de intereses económicos.

Considerando lo expuesto, en las ciencias sociales son diversas las posturas que existen para leer y comprender la ciudad, lo que conduce a una crisis del concepto por la diversidad de elementos propuestos para construir marcos analíticos desde donde estudiarlo (Pereira, 2007). Así, la palabra ciudad, desde el conjunto de disciplinas que reivindican la experiencia subjetiva como forma de acceder al sentido de los fenómenos sociales, gira en torno a toda una serie de construcciones con diferente origen epistemológico que permiten reconocer la ciudad como un producto social y que, en palabras de Souto (1994) y Pérez (2009), es fruto de las experiencias, imágenes, concepciones y percepciones del sujeto.

\section{Ciudad y pedagogía: temas recurrentes}

Con el paso del tiempo y la transformación de las sociedades, la geografía al igual que la enseñanza de la misma han tenido diferentes desarrollos teóricos, metodológicos y prácticos, pasando por diversos enfoques como la geografía biológica orientada a observar el medio natural, o la geografía histórica y la geografía regional desde el positivismo que han sido la base de la orientación tradicional en las escuelas. De acuerdo con Araya (2008), en la enseñanza de la geografía en América Latina "predominan los métodos expositivos y librescos que tienden al verbalismo, a la acumulación de conocimientos y a la excesiva memorización" (p. 2). En tal sentido, los procesos de enseñanza y aprendizaje están alejados de la realidad de la cual hacen parte los estudiantes, es decir, no se inscriben en la cotidianidad y, por tanto, la escuela no contribuye a la formación de un ciudadano consciente de los problemas sociales, de las posibilidades que existen en el territorio y de las formas de intervenir el mundo al cual pertenece.

Considerando lo anterior, las perspectivas actuales de la didáctica de la geografía han planteado la necesidad de pensar en formas alternativas para orientar los procesos de enseñanza, privilegiando un aprendizaje desde los conceptos y el contexto. Para superar una enseñanza descriptiva y memorística, Gurevich, Blanco, Fernández y Tobío (2001) afirman que "los procesos de conceptualización se convierten en los mejores aliados para alcanzar niveles explicativos de la realidad que sean satisfactorios" (p. 21), por ello los conceptos se convierten en un medio para aprender lo social de forma significativa en tanto permiten a los estudiantes conocer e interiorizar las formas como se organizan los conocimientos propios del pensamiento geográfico.

Para Vargas (2009), "un concepto es solo el nombre o una palabra para algo" (p. 31), es decir, que cuando en los procesos de producción de conocimiento se confiere sentido a determinadas palabras nacen conceptos. Así, los mismos solo nombran porciones de conocimiento frente a algo, lo cual implica que se agotan y que por sí solos no puedan explicarlo todo, a la vez que sea necesario recurrir a otros para comprender la realidad. Para Alderoqui (1994), "los conceptos no funcionan nunca de manera aislada, están ligados a otros conceptos que los definen y a su vez colaboran en su definición" (p. 42); por ende, la enseñanza basada en ellos conlleva a que los estudiantes al indagar en su cotidianidad reconozcan las redes de conceptos que se pueden formar para conocer el espacio geográfico.

Al respecto, Rodríguez (2010) explica que los estudiantes pueden formar conceptos a partir de "las experiencias concretas y al relacionar los nuevos a los ya existentes" (p. 62), lo cual conlleva, en un primer momento, a romper los muros del aula para acercar a los estudiantes al territorio y, en segunda instancia, brindar elementos conceptuales que permitan a los jóvenes tejer redes de conceptos para realizar determinadas acciones. Hablar de una enseñanza renovada de la geografía no es una iniciativa 
aislada de un grupo de profesores, es un proyecto académico y pedagógico que busca cambiar los modos como se trabaja la enseñanza de la disciplina en la escuela, a partir de propuestas fundamentadas desde lo teórico, lo metodológico y lo didáctico, por lo que emergen construcciones como las planteadas con anterioridad y que dan sustento a los elementos aquí formulados. Así, la geografía escolar como campo de conocimiento, explica Rodríguez (2002), es

una creación particular y original de la escuela que responde a las finalidades sociales que le son propias, es una de las condiciones básicas que pueden posibilitar una didáctica renovada de la geografía al servicio de la problematización del conocimiento y de la construcción de aprendizajes significativos, funcionales y, en suma, útiles por parte de los alumnos. (p. 184).

\section{Lectura de ciudad en el semillero de geografía para niños}

Una experiencia didáctica que ejemplifica lo planteado sobre el valor educativo de la ciudad en la enseñanza de la geografía la tuvimos en el semillero Geografía para Niños, propuesta didáctica orientada a fortalecer el desarrollo de habilidades espaciales y el conocimiento geográfico de la ciudad de Medellín y el Valle de Aburrá en niños de cuarto y quinto grado de educación básica primaria. A partir del trabajo de cuatro ejes temáticos (ubicación y orientación espacial, manejo de instrumentos de medición de fenómenos climáticos, lectura del paisaje y patrimonio cultural de la ciudad) se procuró que los estudiantes conocieran a través de otros conceptos e instrumentos y tuvieran elementos para leer la ciudad e interactuar con algunos de sus espacios más cercanos.

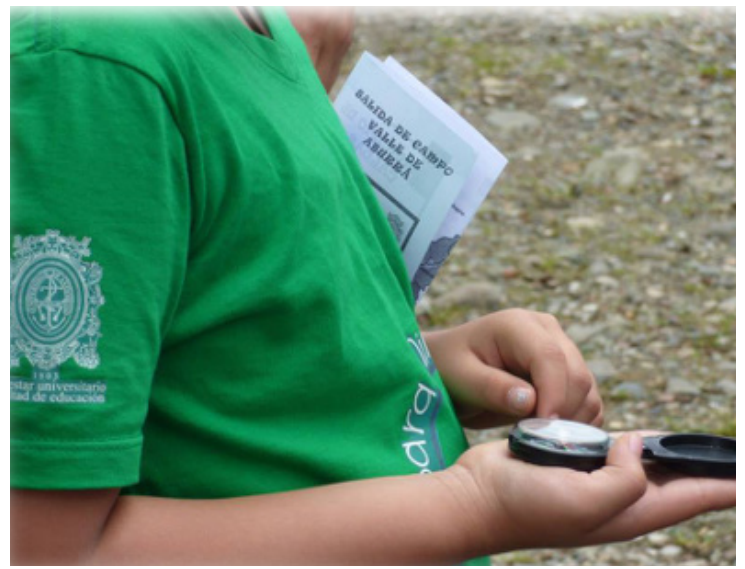

Figura 1. Ejercicio de orientación espacial mediante el uso de la brújula en la ciudad de Medellín.

Fuente: Archivo de los autores.
Para el estudio de la ciudad, como contenido, medio y método de enseñanza de la geografía, se realizaron diferentes actividades en el marco del semillero: la construcción de instrumentos de medición de fenómenos climáticos (veleta, pluviómetro); ejercicios de ubicación y orientación espacial a partir de elementos físicos-naturales como el sol, el viento y la vegetación, y el reconocimiento del patrimonio histórico de la ciudad a partir de un recorrido por el campus principal de la Universidad de Antioquia. Las anteriores actividades se materializaron a través de salidas de campo en las cuales los niños tuvieron la oportunidad de poner en práctica lo aprendido y fortalecer a partir de la experiencia sus conocimientos, haciendo posible que se apropien de la ciudad.

A partir de experiencias como la anterior, teóricos e investigadores de la didáctica de la geografía (Pulgarín, 2010; Castellar, Cavalcanti y Callai, 2012; Fernández, 2007, entre otros) plantean como alternativa al modelo tradicional la enseñanza renovada de la geografía, en la cual destacan la necesidad de enseñar a través de conceptos enmarcados en el contexto de los estudiantes, al igual que pensar procesos que partan de lo más cercano a los sujetos. Desde esta perspectiva, Santiago (2007) afirma que "la contradicción vida cotidiana-aula demanda de nuevas formas de enseñanza" (p. 53), por lo que cobra importancia la ciudad como objeto y categoría de análisis del pensamiento geográfico al ser un espacio cercano a los sujetos, que hace parte de su vida diaria y el cual permite transformar los contenidos curriculares de las ciencias sociales en objetos de enseñanza y aprendizaje vinculados a la cotidianidad.

Asimismo, desde la didáctica de la geografía se establece la importancia de la enseñanza de la ciudad partiendo del para qué pensar lo urbano, tal y como lo plantea Rodríguez (2011). Al tiempo que se piensa en la ciudad de Medellín como medio y objeto didáctico, el espacio urbano actúa como posibilitador de la enseñanza y el aprendizaje de la geografía, de los imaginarios, el saber escolar y la vida cotidiana. Desde los discursos y prácticas de la enseñanza de las ciencias sociales y la geografía se plantea que no es lo mismo el concepto de ciudad en su versión académica y disciplinar que la enseñanza de este. Para Alderoqui (1994), las intenciones y finalidades son distintas, además, considera que al abordar esta categoría en la escuela los profesores deben tener en cuenta "las referencias disciplinares, otras referencias como la edad y las ideas de los alumnos, las prácticas sociales y del mundo social en general" (p. 44), con el propósito de establecer escalas de análisis, el alcance de las actividades, las representaciones de los estudiantes y sus saberes previos.

La riqueza e incorporación de la ciudad en los procesos de enseñanza permite a Moncada (2005) afirmar que "ciudad y educación han sido dos conceptos históricamente inseparables" (p. 38) por las posibilidades de aprendizaje significativo que brinda a maestros y estudiantes. De acuerdo con Pulgarín (2010), la finalidad última de la educación es "formar unos ciudadanos críticos, reflexivos, propositivos y transformadores de su realidad que desarrollen una nueva cultura desde el sentido de pertenencia 
a su territorio" (p. 136), de ahí que la inclusión de la ciudad en el currículo haga énfasis en que los estudiantes observen y vivencien algunos procesos sociales, cambios y permanencias en los espacios, prácticas culturales de la población, y reconozcan las dinámicas económicas y las influencias de las acciones humanas en el espacio (Rodríguez, 2011). Para Moncada (2005),

en la ciudad se construye y se desarrolla el sujeto individual desde su singularidad, desde su particular proyecto de vida y también se construye y desarrolla el sujeto colectivo, como grupo y comunidades humanas que comparten territorios y proyectos comunes. (p. 38).

Así, la ciudad se convierte en una posibilidad para el aprendizaje desde lo vivido, desde la cotidianidad, haciendo explícita la relación del sujeto y el espacio geográfico, dando lugar a que los procesos de enseñanza orientados desde la escuela tengan lugar en un espacio existente, más amplio y concreto.

\section{La ciudad y la práctica pedagógica}

Hablar sobre la necesidad de renovar la enseñanza de la geografía siempre ha sido una tarea más sencilla que hacerlo, ya que las prácticas pedagógicas no cambian al mismo ritmo de las trasformaciones curriculares y los desarrollos investigativos de la didáctica de las ciencias sociales. Además, se deben sortear una serie de barreras institucionales que obstaculizan el desarrollo de algunas propuestas para la enseñanza de geografía en contexto y con-sentido(s) (salidas de campo, enseñanza basada en proyectos, investigación como base del aprendizaje, entre otros). Algunos de estos desafíos son la escasa formación pedagógica y disciplinar de los docentes, la escasez de recursos económicos en el sistema educativo, las condiciones para el trabajo por fuera de la escuela, las condiciones del contexto y las garantías institucionales para el ejercicio de la labor docente.

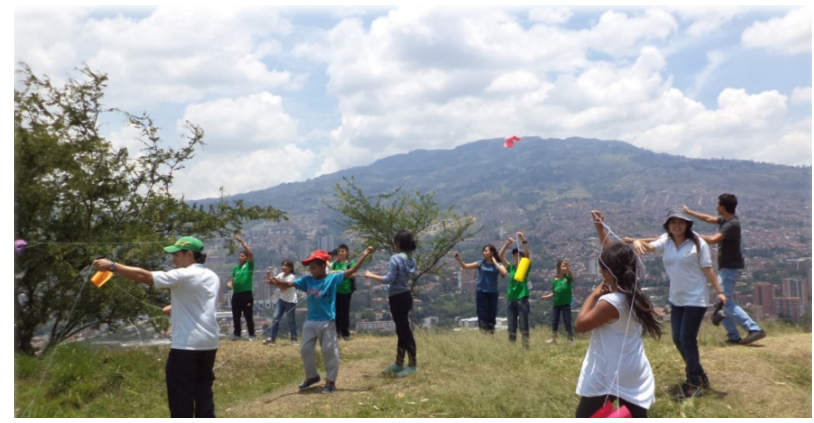

Figura 2. Actividad de elevación de cometas para identificar la influencia de los vientos en el clima en el Valle de Aburrá.

Fuente: Archivo de los autores.
A pesar de ello, se presentan a continuación una serie de elementos a los profesores de ciencias sociales y geografía para pensar la enseñanza de la ciudad a partir de un ejercicio pedagógico del que se desprendan aprendizajes significativos útiles en la cotidianidad de los estudiantes. Los elementos expuestos a continuación pueden ser recreados y ampliados a voluntad de los profesores, con el propósito de hacer énfasis en determinados procesos u objetos que por la realidad de sus estudiantes es pertinente trabajarlos a mayor profundidad.

En primer lugar, la inclusión de la ciudad en el currículo implica romper los muros del aula de clase ${ }^{1} y$ entender que en la ciudad hay otros espacios que también educan: "No solo se educa en la escuela y la familia, también existen otros espacios físicos que lo hacen como la calle, los escenarios donde se practican deportes, los centros comerciales, los parques, museos, restaurantes, entre otros" (Ruíz, 2015, p. 8). En segundo lugar, las tecnologías de la información y de la comunicación son un medio de aprendizaje significativo (Jaramillo, 2014) desde las cuales es posible re/inventar o de/construir las salidas de campo. A partir de herramientas y software se permite a los estudiantes acceder, almacenar, procesar, registrar y compartir información digitalizada que combina texto, imágenes y sonidos, ampliando sus conocimientos en relación con los problemas sociales, económicos, culturales y políticos de las ciudades en las que viven. Así, la proliferación de las TIc en la vida de los niños y los jóvenes debe ser vista como una oportunidad para mostrarles aquellos usos que van más allá de las dinámicas de las redes sociales.

En tercer lugar, la enseñanza renovada de la geografía implica cambiar el tipo de preguntas dirigidas a los estudiantes, deben ser más complejas y no conducir a respuestas que no requieran análisis de información. En este orden de ideas, no es lo mismo preguntar por las principales quebradas de la ciudad de Medellín que por la importancia de las cuencas hídricas de la capital; ambas son preguntas, pero demandan un nivel distinto de análisis de información. En cuarto lugar, crear estrategias de articulación escuela-comunidad facilita la integración de los procesos de enseñanza, de las familias y las instituciones de los entornos más cercanos, de tal manera que sea posible llevar a la escuela los centros de investigación de educación superior, instituciones de salud, producción y de servicios para generar diálogo con esos actores y entender su importancia en las dinámicas de la ciudad.

Los elementos expuestos previamente son una sugerencia a los docentes para que los consideren durante sus ejercicios de planeación y práctica docente. Su concreción es la principal tarea y desafío a partir de la lectura que hacen del contexto en el cual tiene lugar su práctica profesional, al

\footnotetext{
1 Durante las sesiones de trabajo, los docentes y directivos docentes que participaron en este proyecto de investigación manifestaron que el principal temor para implementar las salidas de campo en los procesos de enseńanza son las implicaciones civiles y penales a las que se pueden enfrentar cuando la integridad y seguridad de un estudiante se ve comprometida.
} 
igual que de los aprendizajes que deben lograr los estudiantes al término de su paso por la escuela para responder a las demandas actuales de las sociedades, las cuales se recrean con el paso del tiempo.

\section{Reflexión a modo de cierre}

Pensar la enseñanza renovada de la geografía implica agenciar cambios en la mentalidad y en las prácticas de los docentes. Para ello, es necesario que en muchas ocasiones los maestros piensen y concreten los procesos de enseñanza desde lo más cercano a los sujetos, es decir, a partir del contexto, de las preguntas de los estudiantes, de las representaciones que tienen sobre la realidad, entre otros elementos, para que el maestro desde los contenidos de las ciencias escolares pueda deconstruir la forma como piensa e interviene en la realidad. La enseñanza de la geografía desde la ciudad es solo una de las múltiples posibilidades que existen para mostrar el impacto en el aprendizaje de los estudiantes cuando se parte de las realidades más próximas y cercanas a ellos. La ciudad como concepto y fenómeno con múltiples caras es para el maestro antes que nada un medio y un objeto didáctico, posibilitador de la enseñanza y el aprendizaje del pensamiento geográfico, en el que se reivindica la necesidad de pensar los espacios desde los sujetos que los habitan con el fin de generar sentido de pertenencia, conciencia y transformación del mismo.

Por último, cuando los maestros fundamentan conceptualmente los objetos de conocimiento que enseñan en la escuela, de acuerdo con Pulgarín (2016), reconocen "métodos, técnicas y medios didácticos" (p. 96) que les facilitan pensar y concretar la enseñanza, haciendo posible "el mejoramiento de la práctica docente desde el desarrollo de un aprendizaje con sentido, flexible, pertinente y contextualizado por parte de los estudiantes, como lo requiere la sociedad actual" (Pulgarín, 2016, p. 100). En tal sentido, cobra importancia el acercamiento realizado al concepto de ciudad, puesto que permite comprender la dimensión del concepto y las posibles formas como puede ser usado en la enseñanza de la geografía.

\section{Referencias}

Alderoqui, S. (1994). La ciudad se enseña. En B. Aisenberg y S. Alderoqui (eds.), Didáctica de las ciencias sociales. Aportes y reflexiones (pp. 248-266). Buenos Aires: Paidós.

Alderoqui, S. (2002). Enseñar a pensar la ciudad. En S. Alderoqui y P. Penchansky (eds.), Ciudad y ciudadanos (pp. 33-66). Buenos Aires: Paidós.

Araya, F. (2008). La didáctica de la geografía en América Latina: experiencias y tendencias. Íber. Didáctica de las Ciencias Sociales, Geografía e Historia, (56), 95-110.
Castellar, S., Cavalcanti, L., y Callai, H. (Coords.). (2012). Didática da Geografía. Aportes teóricos e metodológicos. São Paulo: Xama Editora.

Fernández, M. (Ed.). (2007). Geografía y territorios en transformación: nuevos temas para pensar la enseñanza. Buenos Aires: Noveduc.

Gurevich, R., Blanco, J., Fernández, M., y Tobío, O. (2001). Notas sobre la enseñanza de una geografía renovada. Buenos Aires: Aique.

Harvey, D. (1977). Urbanismo y desigualdad social. Madrid: Siglo XXI.

Herce, M. (2013). El negocio del territorio: evolución y perspectivas de la ciudad moderna. Madrid: Alianza.

Jaramillo, Y. (2014). Enseñanza de la geografía desde el estudio de territorios urbanos mediada por aplicaciones web 2.0 (tesis de maestría). Universidad de Antioquia, Medellín, Colombia.

Llorente, M. (Ed.). (2014). Topología del espacio urbano. Palabras, imágenes y experiencias que definen la ciudad. Madrid: Abada Editores.

Moncada, R. (2005). Ciudad, educación y escuela. Educación y ciudad, (7), 35-72.

Pereira, P. (2007). La ciudad: sobre la importancia de nuevos medios para hablar y pensar las ciudades. Mundo Siglo XXI, (9), 69-80.

Pérez, R. (2009). La ciudad, lugar de identidad geográfica y cultural. Fermentum. Revista Venezolana de Sociología y Antropología, 19(54), 35-47.

Pulgarín, R. (2010). Hacia la integración curricular desde el estudio del territorio. En N. Moreno y M. Hurtado (eds.), Itinerarios geográficos en la escuela. Lecturas desde la virtualidad (pp. 133-154.). Bogotá: Geopaideia.

Pulgarín, R. (2016). La geografía escolar: disciplina integradora en la enseñanza de las ciencias. En R. Pulgarín y E. Bernal (eds.), La geografía escondida en sistema educativo nacional (pp. 74-109). Bogotá: Sociedad Geográfica de Colombia.

Rodríguez, E. (2010). Geografía conceptual. Enseñanza y aprendizaje de la geografía en la educación básica secundaria. Bogotá: Estudio Caos.

Rodríguez, E. (2011). ¿Por qué una didáctica de la ciudad latinoamericana? En N. Moreno y A. Cely (eds.), Ciudades leídas y contadas. La ciudad como escenario didáctico para la enseñanza de la geografía (pp. 15-21). Bogotá: Universidad Distrital Francisco José de Caldas. 
Rodríguez, F. (2002). Concebir la geografía escolar desde una nueva perspectiva: una disciplina al servicio de la cultura escolar. Boletín de la Asociación de Geógrafos Españoles, (33), 173-186.

Ruíz, Y. (2015). Espacios no formales en la educación. Divercity un espacio que también educa (informe de investigación). Universidad de Antioquia, Medellín, Colombia.

Santiago, J. (2007). Renovar la enseñanza de la geografía en el mundo actual (tesis de maestría). Universidad de los Andes, San Cristóbal, Venezuela.
Santos, M. (1990). Por una nueva geografía. Madrid: Espasa Universidad.

Souto, X. (1994). Implicaciones didácticas del estudio geográfico de las ciudades. Investigaciones Geográficas, (12), 93-118.

Vargas, G. (2009). Didáctica de la geografía y su aplicación a la enseñanza de la geografía en el tercer ciclo y la enseñanza diversificada de Costa Rica. Revista Educación, 33(1), 75-112. 